\title{
Differing Associations between Optic Nerve Head Strains and Visual Field Loss in Normal- and High-Tension Glaucoma Subjects
}

Thanadet Chuangsuwanich ${ }^{1,2}$, Tin A. Tun ${ }^{3}$, Xiaofei Wang ${ }^{4}$, Zhi Yun Chin ${ }^{1,2}$, Satish Kumar Panda ${ }^{1,2}$, Martin Buist ${ }^{2}$, Nicholas Strouthidis ${ }^{5}$, Shamira Perera ${ }^{3}$, Monisha Nongpiur ${ }^{3,6}$, Tin Aung ${ }^{3,6}$, Michael JA Girard ${ }^{1,6,7}$

${ }^{1}$ Ophthalmic Engineering \& Innovation Laboratory (OEIL), Singapore Eye Research Institute, Singapore National Eye Center, Singapore

${ }^{2}$ Department of Biomedical Engineering, National University of Singapore, Singapore

${ }^{3}$ Singapore Eye Research Institute, Singapore National Eye Centre, Singapore

${ }^{4}$ Laboratory for Biomechanics and Mechanobiology of Ministry of Education, Beijing Advanced

Innovation Center for Biomedical Engineering, School of Biological Science and Medical Engineering,

School of Engineering Medicine, Beihang University, Beijing, China

${ }^{5} \mathrm{NIHR}$ (National Institute of Health Research) Biomedical Sciences Centre, Moorfields Eye Hospital

and UCL Institute of Ophthalmology, London, United Kingdom

${ }^{6}$ Duke-NUS Medical School, Singapore

${ }^{7}$ Institute for Molecular and Clinical Ophthalmology, Basel, Switzerland

Commercial relationship:

Word count:

Tables:

Figures:

Supplementary Material:

Corresponding Author:
None

3756 (manuscript text) 326 (abstract)

1

4

Ophthalmic Engineering \& Innovation Laboratory (OEIL)

Singapore Eye Research Institute (SERI)

The Academia, 20 College Road

Discovery Tower Level 6,

Singapore 169856

https://www.ophthalmic.engineering 


\section{Abstract}

Purpose: To study the associations between optic nerve head (ONH) strains under intraocular pressure (IOP) elevation with retinal sensitivity in glaucoma subjects.

Design: Clinic based cross-sectional study.

Participants: 229 subjects with primary open angle glaucoma (subdivided into 115 high tension glaucoma (HTG) subjects and 114 normal tension glaucoma (NTG) subjects).

Methods: For one eye of each subject, we imaged the $\mathrm{ONH}$ using spectral-domain optical coherence tomography (OCT) under the following conditions: (1) primary gaze and (2) primary gaze with acute IOP elevation (to approximately $33 \mathrm{mmHg}$ ) achieved through ophthalmodynamometry. A 3-dimensional (3D) strain-mapping algorithm was applied to quantify IOP-induced $\mathrm{ONH}$ tissue strain (i.e. deformation) in each $\mathrm{ONH}$. Strains in the pre-lamina tissue (PLT) and the retina, the choroid, the sclera and the lamina cribrosa (LC) were associated (using linear regression) with measures of retinal sensitivity from the 24-2 Humphrey visual field test (Carl Zeiss Meditec, Dublin, CA, USA). This was done globally, then locally according to the regionalization scheme of Garway-Heath et al.

Main Outcome Measures: Associations between $\mathrm{ONH}$ strains and values of retinal sensitivity from visual field testing.

Results: For HTG subjects, we found that (1) there were significant negative linear associations between ONH strains and retinal sensitivity $(p<0.001)$ (on average, a 1\% increase in $\mathrm{ONH}$ strains corresponded to a decrease in retinal sensitivity of $1.1 \mathrm{~dB}$ ), (2) high strain regions co-localized with anatomically-mapped regions of high visual field loss, (3) the strongest negative associations were observed in the superior region 
and in the PLT. In contrast, for NTG subjects, no significant associations between strains and retinal sensitivity were observed except in the supero-temporal region of the LC.

Conclusion: We found significant negative associations between IOP-induced $\mathrm{ONH}$ strains and retinal sensitivity in a relatively large glaucoma cohort. Specifically, HTG subjects who experienced higher ONH strains were more likely to exhibit lower retinal sensitivities. Interestingly, this trend was in general less pronounced in NTG subjects, which could suggest a distinct pathophysiology between the two glaucoma subtypes. 


\section{Introduction}

Loss of vision in glaucoma is preceded by a cascade of cellular events leading to damage of the retinal ganglion cell (RGC) axons. From a biomechanical perspective, the neural and connective tissues around the lamina cribrosa (LC) experience intraocular pressure (IOP)-related stresses and strains; these could in turn elicit RGC damage (directly or indirectly) at the level of the LC. ${ }^{1}$ Apart from IOP, the biomechanical stresses in the LC can also be influenced by age, ${ }^{2}$ the presence of ocular comorbidities (e.g. myopia) ${ }^{3}$ and a fluctuating cerebrospinal fluid pressure (CSFP). ${ }^{4}$ Increased biomechanical stresses due to the aforementioned factors (and their interactions) could ultimately influence an already fragile $\mathrm{ONH}^{5}$, and the resulting deformations could correlate with functional loss in glaucoma. Using OCT imaging and computational algorithms, several authors have successfully reported IOP-induced biomechanical deformations within the ONH tissues in terms of 'strains ${ }^{\prime 6-9}$, which can in-turn be used for calculations of tissue stiffness/compliance ${ }^{10}$. Apart from our earlier study ${ }^{11}$ which reported that the amount of $\mathrm{ONH}$ strains alleviated due to trabeculectomy was associated with the extent of visual field loss based on a few glaucoma subjects, no studies have investigated associations between local $\mathrm{ONH}$ strains and visual field loss in glaucoma. We believe that such associations would provide strong evidence to support the clinical usefulness of $\mathrm{ONH}$ strains extracted from OCT images for both glaucoma diagnosis and prognosis.

In addition, with the high prevalence of normal tension glaucoma (NTG), especially in Asian populations ${ }^{12,13}$, we believe that it would be valuable to compare the aforementioned associations between NTG and high-tension glaucoma (HTG) subjects. The differences in relationships between IOP-induced strain and functional 
loss in glaucoma in NTG and HTG subjects can possibly highlight differences in etiologies, if they exist, between the two glaucoma subgroups.

The aim of this study was to investigate the relationship between in vivo local ONH strains (induced by IOP elevation) and retinal sensitivity values measured from 24-2 Humphrey visual field tests in NTG and HTG subjects. To this end, we applied a strain/displacement mapping algorithm, based on our previous works ${ }^{6,7,9,14}$, to optical coherence tomography (OCT) images to quantify in vivo $\mathrm{ONH}$ strains in each subject. We then utilized a map derived by Garway-Heath et al. ${ }^{15}$ to co-localize anatomical $\mathrm{ONH}$ regions to their corresponding visual field counterparts.

\section{Methods}

Our goal was to quantitively map 3D ONH strains in NTG and HTG subjects under IOP elevation and correlate the $\mathrm{ONH}$ strains to the retinal sensitivity values obtained from the 24-2 Humphrey visual field test. To this end, we first imaged each subject's $\mathrm{ONH}$ in baseline gaze using OCT, and subsequently, under acute IOP elevation. $\mathrm{ONH}$ tissue deformations were mapped using a digital volume correlation (DVC) algorithm applied to the pair of OCT volumes. Such deformations were then statistically correlated to retinal sensitivity values using linear regressions across the entire $\mathrm{ONH}$ tissues and across regions of the visual field using a spatial map provided by Garway Heath et al. ${ }^{15}$ Below is a detailed description of our methodology.

\section{Subjects Recruitment}

We recruited 229 subjects with primary open angle glaucoma (115 subjects with HTG and 114 with NTG) from glaucoma clinics at the Singapore National Eye Centre. We included subjects aged more than 50 years old, of Chinese ethnicity 
(predominant in Singapore), with a refractive error of \pm 3 diopters, and excluded subjects who underwent prior intraocular/orbital/brain surgeries, subjects with past history of strabismus, ocular trauma, ocular motor palsies, orbital/brain tumors; with clinically abnormal saccadic or pursuit eye movements; subjects with poor LC visibility in OCT (<50\% en-face visibility); subjects with known carotid or peripheral vascular disease; or with any other abnormal ocular conditions. Glaucoma was defined as glaucomatous optic neuropathy, characterized as loss of neuroretinal rim with vertical cup-to-disc ratio $>0.7$ or focal notching with nerve fiber layer defect attributable to glaucoma and/or asymmetry of cup-to disc ratio between eyes $>0.2$, with repeatable glaucomatous visual field defects (independent of the IOP value) in at least 1 eye. NTG subjects had consistently low/normal IOP $(<21 \mathrm{mmHg})$ before treatment in the study eye; HTG subjects had elevated IOP $(>21 \mathrm{mmHg})$ before treatment in the study eye.

Each subject underwent the following ocular examinations: (1) measurement of refraction using an autokeratometer (RK-5; Canon, Tokyo, Japan); (2) measurement of axial length, central corneal thickness (CCT) and anterior chamber depth (ACD) using a commercial device (Lenstar LS 900; Haag-Streit AG, Switzerland). For each tested eye we performed a visual field test using a standard achromatic perimetry with the Humphrey Field Analyser (Carl Zeiss Meditec, Dublin, CA).

This study was approved by the SingHealth Centralized Institutional Review Board and adhered to the tenets of the Declaration of Helsinki. Written informed consent was obtained from each subject.

\section{Visual Field Testing}

Subjects with visual field deficits related to diabetic retinopathy or any other optic neuropathies, or advanced glaucoma precluding acute elevation of IOP were 
excluded. Unreliable visual field test results with a false-positive error $>15 \%$, or a fixation loss $>33 \%$ were excluded ${ }^{16}$. Thresholding Algorithm (Swedish Interactive Testing Algorhithm) standard 24-2 program were selected. For every selected subject the retinal sensitivity map from the Humphrey Visual Field report was used in our study.

\section{OCT Imaging}

We selected one eye at random for each subject and we imaged the $\mathrm{ONH}$ with spectral-domain OCT (Spectralis; Heidelberg Engineering GmbH, Heidelberg, Germany). The imaging protocol was similar to that from our previous work. ${ }^{6}$ In brief, we conducted a raster scan of the $\mathrm{ONH}$ (covering a rectangular region of $15^{\circ} \times 10^{\circ}$ centered at the $\mathrm{ONH}$ ), comprising of 97 serial B-scans, with each B-scan comprising of 384 A-scans (Figure 1a). The average distance between B-scans was $35.1 \mu \mathrm{m}$ and the axial and lateral B-scan pixel resolution were on average $3.87 \mu \mathrm{m}$ and $11.5 \mu \mathrm{m}$ respectively. All A-scans were averaged 10 times during acquisition to reduce speckle noise. Each eye was scanned two times under two conditions - baseline OCT position, and acute IOP elevation. Each subject was administered with $1.0 \%$ Tropicamide to dilate the pupils before imaging.

\section{OCT imaging during acute IOP elevation}

For each eye in baseline position, we applied a constant force of $0.65 \mathrm{~N}$ to the temporal side of the lower eyelid using an opthalmodynamometer, as per a wellestablished protocol. 6,7 This force raised IOP to about $35 \mathrm{mmHg}$ and was maintained constant. IOP was then re-assessed with a Tono-Pen (Reichert Instruments GmbH, Munich, Germany) and the ONH was imaged with OCT in baseline position.

\section{ONH Reconstruction through Automatic Segmentation}


For each $\mathrm{ONH}$, we automatically segmented the following tissue groups - the PLT+retina, the choroid, the visible portion of the peripapillary sclera, and the visible portion of the LC (Figure 1b). This was done using a deep-learning algorithm similar to that designed in our previous work. ${ }^{17}$ Here, segmentation was required so as to summarize strains for any chosen tissue group in subsequent steps. BMO points were also automatically extracted with a custom algorithm. Note that BMO points lie within a plane (the BMO plane), and such a plane can be used as a horizontal reference plane for each $\mathrm{ONH}$.

\section{ONH Regional Division}

To ensure un-biased comparisons between groups (NTG vs HTG) for 3D deformations/strains, we first limited our en-face field-of-view to a region of $2800 \mathrm{x}$ $2800 \mu \mathrm{m}^{2}$ centered on the BMO center for all ONHs (Figure 1c). Each ONH was further divided into six regions (nasal, supero-nasal, supero-temporal, temporal, inferotemporal and infero-nasal) according to the regionalization scheme of Garway-Heath et al. ${ }^{15}$ (Figure 1c). To aid in calculation of strains, we defined a cylindrical coordinate system with the BMO center as the origin and the axes are defined as the radial distance $(r)$, the circumferential angle $(\Theta)$, and the axial distance ( $z$; depth) according to Figure 1c-d.

Each region of the $\mathrm{ONH}$ was spatially associated to the $24-2$ visual field test points according to the Garway-Heath scheme shown in Figure 1e.

\section{In Vivo Displacement and Strain Mapping of the ONH}

We used a commercial DVC module (Amira, 2020.3, Waltham, Massachussets: Thermo Fisher Scientific) to map the three-dimensional deformations between the baseline scan and acute IOP elevation scan for each patient. The working principle of this commercial DVC module is similar to our prior DVC implementation ${ }^{9}$, albeit with 
an improved speed efficiency. Details of the DVC algorithm used in this study is provided in Appendix A. Briefly, each ONH morphology was sub-divided into $\sim 4,000$ cubic elements, and $\sim 3,500$ nodes (points), at which locations 3D displacements (vectors) were mapped following an acute IOP elevation. We then derived the (1) effective strain, (2) circumferential strain, and (3) axial strain from the 3D displacements. The effective strain is a convenient local measure of $3 \mathrm{D}$ deformation that takes into account both the compressive and tensile effects. In other words, the higher the compressive or tensile strain, the higher the effective $\operatorname{strain}^{6,} 9,14$ Circumferential strain takes into account the circular geometry of the eye and it highlights the effect of the circumferential forces (i.e., hoop stress) borne by the scleral canal under IOP elevation. ${ }^{18}$ Axial strain corresponds to the axial direction of the eye and it highlights the $\mathrm{ONH}$ tissue compression under IOP elevation. Details of the strain derivation is provided in Appendix B, and further validation of the DVC and its effects on strain in Appendix C.

\section{Statistical Analysis}

To study the associations between IOP-induced strain and retinal sensitivity, we calculated the mean values of $\mathrm{ONH}$ effective strain (all tissues and all regions) and the mean values of retinal sensitivity for each subject. We then fit a linear regression model using MATLAB for each subject group with the effective strain as a dependent variable and the retinal sensitivity as an independent variable. We also performed similar analyses separately for the PLT+Retina, Choroid, Sclera and LC.

To study the regional association of $\mathrm{ONH}$ effective strain and retinal sensitivity, we calculated the mean values of effective strain in each region and their respective mean values of retinal sensitivity for each subject. In essence, each subject had 6 data points (corresponding to mean effective strain and mean retinal sensitivity in each 
region) and we fit a linear regression model on each subject. To represent an average regional association across all subjects, we also fit a linear regression model on all data points across every subject of each group.

To investigate the association of each tissue, each region, and each subject group to each type of strain (effective strain, circumferential strain, and axial strain), we performed in total 142 linear fits (number of combinations of the aforementioned parameters). We performed a Bonferroni correction to adjust the statistically significant $\mathrm{p}$-value to the number of comparisons and reported significant associations based on the adjusted p-value.

Statistical significance level was set at 0.05 for all linear model fits. We compared the strength of the linear relationships based on the estimated coefficients.

\section{Results}

\section{Demographics and IOP elevation}

A total of 229 Chinese subjects were recruited (consisting of 115 subjects with HTG and 114 with NTG). We excluded 24 HTG subjects and 23 NTG subjects from the study due to a low en-face LC visibility $(<50 \%$ of the BMO area) and/or unreliable visual field results. Therefore, 91 HTG subjects and 91 NTG subjects were included in the final analysis. Out of 91 HTG subjects, 32 subjects were female. Out of 91 NTG subjects, 41 subjects were female.

There were no significant differences ( $p>0.05$ ) across both groups in terms of age [HTG: $69 \pm 5, \mathrm{NTG:} 67 \pm 6]$, systolic blood pressure [HTG: $141 \pm 16 \mathrm{mmHg}, \mathrm{NTG}$ : $140 \pm 20 \mathrm{mmHg}$ ], diastolic blood pressure [HTG: $75 \pm 9 \mathrm{mmHg}, \mathrm{NTG:} 74 \pm 9 \mathrm{mmHg}$ ], axial length [HTG: $24.2 \pm 1.0 \mathrm{~mm}, \mathrm{NTG}: 24.4 \pm 1.0 \mathrm{~mm}$ ], visual field mean deviation [HTG: $-7.54 \pm 5.05 \mathrm{~dB}, \mathrm{NTG}:-6.56 \pm 4.91 \mathrm{~dB}$ ], pattern standard deviation [HTG: 7.18 
$\pm 3.79 \mathrm{~dB}, \mathrm{NTG}: 7.22 \pm 3.05 \mathrm{~dB}$ ], baseline IOP (on the day of the experiment) [HTG: $17.3 \pm 2.9 \mathrm{mmHg}, \mathrm{NTG}: 16.0 \pm 2.5 \mathrm{mmHg}$ ], and IOP during ophthalmodynamometer indentation [HTG: $34.5 \pm 7.0 \mathrm{mmHg}, \mathrm{NTG}: 34.8 \pm 6.5 \mathrm{mmHg}$.

In terms of visual field, there were no significant differences ( $p>0.05)$ across both groups in terms of MD values [HTG: $-7.46 \pm 5.9 \mathrm{~dB}$, NTG: $-6.17 \pm 5.8 \mathrm{~dB}$ ], mean retinal sensitivity values [HTG: $21.54 \pm 4.6 \mathrm{~dB}$, NTG: $19.43 \pm 9.40 \mathrm{~dB}$ ] and mean pattern deviation values [HTG: $-5.51 \pm 5.61 \mathrm{~dB}$, NTG: $-5.13 \pm 5.63 \mathrm{~dB}$ ].

\section{Significant Negative Associations between Effective Strain and Retinal Sensitivity in HTG Subjects}

Across the entire $\mathrm{ONH}$ (all tissues, all regions), we found a significant negative association between the average retinal sensitivity $(\mathrm{dB})$ and the average effective strain for HTG subjects $(p<0.001)$ with a regression coefficient $(\beta)$ of -1.1 (Figure $2 a)$. In other words, a $1 \%$ increase in effective strain correlate to $1.1 \mathrm{~dB}$ decrease in retinal sensitivity.

For NTG subjects, we found no significant association $(p=0.4)$ between the average retinal sensitivity $(\mathrm{dB})$ and the average effective strain (Figure $\mathbf{2} \mathbf{b}$ ).

\section{Strongest Negative Association in the PLT Tissue of HTG subjects}

For all ONH tissues (LC, PLT, Choroid and sclera - Figure 3a-d), we found significant negative associations between the average retinal sensitivity $(\mathrm{dB})$ and the average effective strain for HTG subjects (all tissues p-values <0.001). The tissues exhibiting the strongest negative associations for HTG subjects were the PLT $(\beta=-$ 1.1), followed by the sclera $(\beta=-1.0)$, the $\operatorname{LC}(\beta=-0.9)$ and the choroid $(\beta=-0.9)$. 
For NTG subjects, there were no significant associations between the average retinal sensitivity $(\mathrm{dB})$ and the average effective strain for all tissues $(p>0.05$, Figure 3a-d).

\section{ONH Regions with High Effective Strains Corresponds to Regions with Low Retinal Sensitivity}

Across all HTG and NTG subjects, there is a significant association between regions with high strains and regions with low retinal sensitivities $(p<0.001$ and $p=$ 0.004 respectively, Figure 4a).

To illustrate this association, Figure $\mathbf{4 b}$ shows a co-localization of the highest average strain value (11.3\%) and the lowest average retinal sensitivity value (13 $\mathrm{dB})$ in the superior-nasal region of an HTG subject. Likewise, Figure 4c shows a colocalization of the highest average strain value (4.1\%) and the lowest average retinal sensitivity value $(22 \mathrm{~dB})$ in the inferior-temporal region of an NTG subject.

\section{Ranking of all Associations}

There were 6 significant linear associations (after Bonferroni correction) between each type of strain and the retinal sensitivity from a total of 142 associations (Table 1). All significant associations were negative (i.e., higher strains correspond to lower retinal sensitivities). The strongest association for HTG subjects was observed in the superior-temporal region of the PLT tissue with circumferential strain as a measure, with a $1 \%$ increase in circumferential strain corresponding to approximately $3.65 \mathrm{~dB}$ reduction in retinal sensitivity.

We found a single significant association for NTG subjects, specifically in the superior-temporal region of the LC tissue with circumferential strain as a measure, with a $1 \%$ increase in circumferential strain corresponding to approximately $2.74 \mathrm{~dB}$ reduction in retinal sensitivity. 
All significant associations were observed in the superior region, with 5 of them occurring in the supero-temporal region and one in the supero-nasal region.

\section{Discussion}

In this study, we investigated the associations between IOP-induced $\mathrm{ONH}$ strain and retinal sensitivity, with respect to each $\mathrm{ONH}$ tissue and each $\mathrm{ONH}$ region. We also compared the associations across two group of glaucoma subjects (NTG and HTG). For HTG subjects, we found significant negative associations between $\mathrm{ONH}$ strains induced by IOP elevation and retinal sensitivity; whereas for NTG subjects, such associations were less significant.

We found that IOP-induced ONH effective strain had a significant association with retinal sensitivity in HTG subjects (Figure 2). This association has been reported before in our earlier study, ${ }^{14}$ however, the key differences were that (1) we reported associations with respect to IOP lowering (from trabeculectomy), whereas herein, with respect to IOP elevation (through ophthalmo-dynamometry); (2) our population was significantly larger (229 glaucoma subjects herein vs 9 glaucoma subjects in our previous study). In addition, we found that the association was negative, and on average an increase of $1 \%$ in $\mathrm{ONH}$ effective strains corresponded to a decrease of 1.1 $\mathrm{dB}$ in retinal sensitivity. Interestingly, significant negative associations were found for any given tissue, whether neural or connective (Figure 3). According to the biomechanical theory of glaucoma, ${ }^{19}$ IOP-induced strain could potentially: (1) directly induce mechanical damage to the $\mathrm{RGCs}^{20},(2)$ disrupt the microcapillary blood flow at the LC, choroid and the retina ${ }^{21-23}$ and (3) disrupt the axoplasmic flow ${ }^{24}$. Therefore, it would be plausible that eyes with structurally weaker ONHs (and thus higher strains) would be more susceptible to visual field loss. The results from this study suggest that a simple mechanical test (IOP elevation via ophthalmodynamometer) could provide 
insights into the structural-functional relationship for glaucoma subjects. Further studies should be carried out to investigate whether such relationships could be used to predict glaucoma progression.

Of note, we observed no global associations between $\mathrm{ONH}$ strains and retinal sensitivity in NTG subjects (Figure 2) on average for all ONH tissues nor for each specific $\mathrm{ONH}$ tissue. This is an interesting finding since all HTG and NTG subjects were matched demographically and both groups had similar visual field indices. On the surface, this could imply that IOP elevation (or its fluctuation) may not influence ONH biomechanics in NTG subjects in a way that directly translates to visual field loss. It may support the notion of a different aetiology for NTG subjects that is IOP independent. ${ }^{25,} 26$ For instance, several factors have been proposed to explain the development and progression of glaucoma at lower levels of IOP, such as a vascular deficiency, ${ }^{25}$ a low CSFP, ${ }^{27,} 28$ structural weaknesses of ocular tissues ${ }^{29,} 30$, or the presence of a strong optic nerve head traction force during eye movements. ${ }^{6,31-34}$

In terms of regional associations, we observed that regions of high strains were co-localized with anatomically-mapped regions of high visual field loss for both groups of subjects, with a stronger association observed in HTG subjects (Figure 4a). In glaucoma, the superior and inferior region of the optic nerve are more susceptible to ganglion cell loss, ${ }^{35}$ which gives rise to what is described as an hourglass-like pattern of neuronal death. This follows the anatomical feature of the supportive collagenous beams at the LC, where the beams are less dense (resulting in larger optic nerve passages) in the superior and inferior regions as compared to the nasal and temporal region. ${ }^{36}$ It is likely that the superior and inferior regions of the LC are more susceptible to IOP-related forces (resulting in higher deformation and strains) due to the lesser presence of the supporting collagenous beams. In addition, the remodelling process 
of the connective tissue during glaucoma development may also contribute to the focal damage to the nerve cells, as this process could change the LC pores diameters, the collagen beam thickness and other LC morphologies, all of which influence the LC responses' to IOP elevation. ${ }^{37}$ In our study, we observed that the superior regions (both superior-nasal and superior-temporal) of the $\mathrm{ONH}$ had the strongest associations (Table 1). Although our study agrees with the aforementioned focal pattern neuronal loss in the superior regions, we did not find significant association in the inferior regions, which are also the common site for $\mathrm{ONH}$ damage in glaucoma. ${ }^{38-}$ 40 This discrepancy may be attributed to the different stages of glaucoma disease as remodelling of the extracellular matrix can lead to a rapid change of $\mathrm{ONH}$ tissues responses to a mechanical stress. Our subjects consist of a broad spectrum of glaucoma severity (from mild to severe) and it is possible that the inferior-temporal structural weaknesses may be more prominent in a high severity glaucoma. Of note, we observed a significant association in NTG subjects in the superior-temporal region of the LC tissue (Table 1).

In terms of strains measures, circumferential strains provided the strongest associations (Table 1). Circumferential ONH strains can primarily be attributed to IOPrelated stresses acting on the peripapillary sclera ${ }^{19}$, this latter being supported by a circumferentially-arranged network of collagen and elastin fibres. ${ }^{41}$ Circumferential $\mathrm{ONH}$ strains can be thought of as local measures of scleral canal expansion, ${ }^{42,43}$ where higher circumferential strains may imply that the ONH's ability to withstand IOPrelated forces was compromised, possibly from the weakening of the collagenous ring surrounding the optic disc. ${ }^{44}$ Therefore, it would be sensible for circumferential strains to be strongly associated with visual field loss as observed in this study. Tissue-wise, the PLT provided the strongest association. The significant thinning of the PLT tissue 
in glaucoma disease, resulting from the loss of a wide variety of cells within the retina, including ganglion cells, muller cells and others, ${ }^{45}$ may compromise the ability of the PLT to tolerate IOP-induced forces causing an increase (that corresponds to the severity of glaucoma disease) in IOP-induced strains in the PLT.

Several limitations in this study warrant further investigation. First, we employed a simple linear regression to link strain to measures of visual field loss. However, this relationship could be highly non-linear. Second, we did not categorize glaucoma into different stages. Since different stages of glaucoma could have distinct biomechanical manifestations, ${ }^{46-48}$ our analysis would miss out on those differences and instead represent an average trend of all glaucoma stages. Third, our definition of NTG vs HTG (based on an IOP value of $21 \mathrm{mmHg}$ ) introduces an additional degree of uncertainties with respect to our analysis for those subjects with IOP values of $\sim 21$ $\mathrm{mmHg}$ before treatment. However, this definition is still the standard for classifying NTG patients. ${ }^{49-51}$ Fourth, this study was cross-sectional and therefore does not provide insights into the causal relationship between strains and visual field loss. A prospective study is warranted. Fifth, our study was limited to subjects of Chinese ethnicity and may thus not be representative of other populations. Sixth, errors in both displacement and strain could occasionally occur. The errors observed here could arise from various sources such as OCT registration errors (intrinsic to the OCT machine), rotation of subjects' head during OCT acquisition, OCT speckle noise and IOP fluctuations from ocular pulsations, ${ }^{52}$ all of which were difficult to control. However, displacement error magnitudes were still lower than the OCT voxel resolution (Appendix C); thus, these errors should not have significantly affected the observed trends. 
In conclusion, we found that there were significant negative associations between IOP-induced ONH strains and retinal sensitivity. In short, HTG subjects who experienced higher strains due to acute IOP elevation also exhibited lower retinal sensitives. We also identified the tissue (PLT) and the ONH regions (Superior regions) where these associations were the strongest. In addition, weaker associations were observed in NTG subjects, which could be indicative of differing aetiologies between the NTG and HTG groups.

\section{Acknowledgements}

Acknowledgement is made to (1) the donors of the National Glaucoma Research, a program of the BrightFocus Foundation, for support of this research (G2021010S [MG]), (2) the Singapore Ministry of Education, Academic Research Funds, Tier 2 (R397-000-280-112; R-397-000-308-112 [MG]) \& Tier 1 (R-397-000-294-114 [MG]), (3) the "Retinal Analytics through Machine learning aiding Physics (RAMP)" project supported by the National Research Foundation, Prime Minister's Office, Singapore under its Intra-Create Thematic Grant "Intersection Of Engineering And Health" NRF2019-THE002-0006 awarded to the Singapore MIT Alliance for Research and Technology (SMART) Centre, and (4) the National Natural Science Foundation of China (12002025 [XW]). 


\section{References}

1. Anthony J. Bellezza RTH, Claude F. Burgoyne. The optic nerve head as a biomechanical structure: Initial finite element modeling. Investigative Ophthalmology \& Visual Science 2000;41(10):2991-3000.

2. Liu B, McNally S, Kilpatrick JI, et al. Aging and ocular tissue stiffness in glaucoma. Survey of ophthalmology 2018;63(1):56-74.

3. Miki A, Ikuno Y, Asai T, et al. Defects of the lamina cribrosa in high myopia and glaucoma. PloS one 2015;10(9):e0137909.

4. Feola AJ, Coudrillier B, Mulvihill J, et al. Deformation of the lamina cribrosa and optic nerve due to changes in cerebrospinal fluid pressure. Investigative ophthalmology \& visual science $2017 ; 58(4): 2070-8$.

5. Sigal IA, Flanagan JG, Ethier CR. Factors influencing optic nerve head biomechanics. Investigative ophthalmology \& visual science 2005;46(11):4189-99.

6. Wang $X$, Beotra MR, Tun TA, et al. In vivo 3-dimensional strain mapping confirms large optic nerve head deformations following horizontal eye movements. Investigative ophthalmology \& visual science 2016;57(13):5825-33.

7. Beotra MR, Wang $X$, Tun TA, et al. In vivo three-dimensional lamina cribrosa strains in healthy, ocular hypertensive, and glaucoma eyes following acute intraocular pressure elevation. Investigative ophthalmology \& visual science 2018;59(1):260-72.

8. Girard MJ, Beotra MR, Chin KS, et al. In Vivo 3-Dimensional Strain Mapping of the Optic Nerve Head Following Intraocular Pressure Lowering by Trabeculectomy. Ophthalmology 2016;123(6):1190-200.

9. Girard MJ, Strouthidis NG, Desjardins A, et al. In vivo optic nerve head biomechanics: performance testing of a three-dimensional tracking algorithm. Journal of The Royal Society Interface 2013;10(87):20130459.

10. Zhang L, Thakku SG, Beotra MR, et al. Verification of a virtual fields method to extract the mechanical properties of human optic nerve head tissues in vivo. Biomech Model Mechanobiol 2017;16(3):871-87.

11. Girard MJ, Beotra MR, Chin KS, et al. In Vivo 3-Dimensional Strain Mapping of the Optic Nerve Head Following Intraocular Pressure Lowering by Trabeculectomy. Ophthalmology 2016.

12. Kim C-s, Seong GJ, Lee N-h, et al. Prevalence of primary open-angle glaucoma in central South Korea: the Namil study. Ophthalmology 2011;118(6):1024-30.

13. Iwase A, Suzuki Y, Araie M, et al. The prevalence of primary open-angle glaucoma in Japanese: the Tajimi Study. Ophthalmology 2004;111(9):1641-8.

14. Girard MJ, Beotra MR, Chin KS, et al. In vivo 3-dimensional strain mapping of the optic nerve head following intraocular pressure lowering by trabeculectomy.

Ophthalmology 2016;123(6):1190-200.

15. Garway-Heath DF, Poinoosawmy D, Fitzke FW, Hitchings RA. Mapping the visual field to the optic disc in normal tension glaucoma eyes. Ophthalmology 2000;107(10):1809-15. 16. Ding X, Chang RT, Guo X, et al. Visual field defect classification in the Zhongshan Ophthalmic Center-Brien Holden Vision Institute High Myopia Registry Study. British Journal of Ophthalmology 2016:bjophthalmol-2015-307942. 
17. Devalla SK, Renukanand PK, Sreedhar BK, et al. DRUNET: a dilated-residual U-Net deep learning network to segment optic nerve head tissues in optical coherence tomography images. Biomedical optics express 2018;9(7):3244-65.

18. Downs JC, Roberts MD, Burgoyne CF. The mechanical environment of the optic nerve head in glaucoma. Optometry and vision science: official publication of the American Academy of Optometry 2008;85(6):425.

19. Strouthidis NG, Girard MJ. Altering the way the optic nerve head responds to intraocular pressure-a potential approach to glaucoma therapy. Current opinion in pharmacology 2013;13(1):83-9.

20. Guo L, Moss SE, Alexander RA, et al. Retinal ganglion cell apoptosis in glaucoma is related to intraocular pressure and IOP-induced effects on extracellular matrix. Investigative ophthalmology \& visual science 2005;46(1):175-82.

21. Chuangsuwanich T, Hung PT, Wang $X$, et al. Morphometric, Hemodynamic, and Biomechanical Factors Influencing Blood Flow and Oxygen Concentration in the Human Lamina Cribrosa. Investigative ophthalmology \& visual science 2020;61(4):3-.

22. Chuangsuwanich T, Birgersson KE, Thiery A, et al. Factors Influencing Lamina Cribrosa Microcapillary Hemodynamics and Oxygen Concentrations. Investigative Ophthalmology \& Visual Science 2016;57(14):6167-79.

23. Geijer C, Bill A. Effects of raised intraocular pressure on retinal, prelaminar, laminar, and retrolaminar optic nerve blood flow in monkeys. Investigative ophthalmology \& visual science 1979;18(10):1030-42.

24. Anderson DR, Hendrickson A. Effect of intraocular pressure on rapid axoplasmic transport in monkey optic nerve. Investigative ophthalmology \& visual science 1974;13(10):771-83.

25. Yamamoto T, Kitazawa Y. Vascular pathogenesis of normal-tension glaucoma: a possible pathogenetic factor, other than intraocular pressure, of glaucomatous optic neuropathy. Progress in retinal and eye research 1998;17(1):127-43.

26. Mudumbai RC. Clinical update on normal tension glaucoma. Seminars in Ophthalmology: Taylor \& Francis, 2013; v. 28.

27. Lee SH, Kwak SW, Kang EM, et al. Estimated trans-lamina cribrosa pressure differences in low-teen and high-teen intraocular pressure normal tension glaucoma: the Korean National Health and Nutrition Examination Survey. PloS one 2016;11(2):e0148412.

28. Chen BH, Drucker MD, Louis KM, Richards DW. Progression of normal-tension glaucoma after ventriculoperitoneal shunt to decrease cerebrospinal fluid pressure. Journal of glaucoma 2016;25(1):e50-e2.

29. Park JH, Jun RM, Choi K-R. Significance of corneal biomechanical properties in patients with progressive normal-tension glaucoma. British Journal of Ophthalmology 2015;99(6):746-51.

30. Kim YC, Koo YH, Jung KI, Park CK. Impact of posterior sclera on glaucoma progression in treated myopic normal-tension glaucoma using reconstructed optical coherence tomographic images. Investigative ophthalmology \& visual science 2019;60(6):2198-207. 31. Chang MY, Shin A, Park J, et al. Deformation of optic nerve head and peripapillary tissues by horizontal duction. American journal of ophthalmology 2017;174:85-94.

32. Demer JL. Optic nerve sheath as a novel mechanical load on the globe in ocular duction. Investigative Ophthalmology \& Visual Science 2016;57(4):1826-38. 
33. Demer JL, Clark RA, Suh SY, et al. Magnetic resonance imaging of optic nerve traction during adduction in primary open-angle glaucoma with normal intraocular pressure. Investigative ophthalmology \& visual science 2017;58(10):4114-25.

34. Demer JL, Clark RA, Suh SY, et al. Optic nerve traction during adduction in open angle glaucoma with normal versus elevated intraocular pressure. Current eye research 2020;45(2):199-210.

35. Quigley HA. Neuronal death in glaucoma. Progress in retinal and eye research 1999;18(1):39-57.

36. Nadler Z, Wang B, Schuman JS, et al. In vivo three-dimensional characterization of the healthy human lamina cribrosa with adaptive optics spectral-domain optical coherence tomography. Investigative ophthalmology \& visual science 2014;55(10):6459-66.

37. Wang B, Nevins JE, Nadler Z, et al. In vivo lamina cribrosa micro-architecture in healthy and glaucomatous eyes as assessed by optical coherence tomography. Investigative ophthalmology \& visual science 2013;54(13):8270-4.

38. Fazio MA, Grytz R, Bruno L, et al. Regional variations in mechanical strain in the posterior human sclera. Investigative ophthalmology \& visual science 2012;53(9):5326-33.

39. Kamal D, Viswanathan A, Garway-Heath D, et al. Detection of optic disc change with the Heidelberg retina tomograph before confirmed visual field change in ocular hypertensives converting to early glaucoma. British Journal of Ophthalmology 1999;83(3):290-4.

40. Strouthidis NG, Gardiner SK, Sinapis C, et al. The spatial pattern of neuroretinal rim loss in ocular hypertension. Investigative ophthalmology \& visual science 2009;50(8):373742.

41. Grytz R, Meschke G, Jonas JB. The collagen fibril architecture in the lamina cribrosa and peripapillary sclera predicted by a computational remodeling approach. Biomechanics and modeling in mechanobiology 2011;10(3):371-82.

42. Sigal IA, Yang H, Roberts MD, et al. IOP-induced lamina cribrosa displacement and scleral canal expansion: an analysis of factor interactions using parameterized eye-specific models. Investigative ophthalmology \& visual science 2011;52(3):1896-907.

43. Coudrillier B, Boote C, Quigley HA, Nguyen TD. Scleral anisotropy and its effects on the mechanical response of the optic nerve head. Biomechanics and modeling in mechanobiology 2013;12(5):941-63.

44. Braunsmann C, Hammer CM, Rheinlaender J, et al. Evaluation of lamina cribrosa and peripapillary sclera stiffness in pseudoexfoliation and normal eyes by atomic force microscopy. Investigative ophthalmology \& visual science 2012;53(6):2960-7.

45. Yang H, Downs JC, Bellezza A, et al. 3-D histomorphometry of the normal and early glaucomatous monkey optic nerve head: prelaminar neural tissues and cupping. Investigative ophthalmology \& visual science 2007;48(11):5068-84.

46. Park SC, Brumm J, Furlanetto RL, et al. Lamina cribrosa depth in different stages of glaucoma. Investigative ophthalmology \& visual science 2015;56(3):2059-64.

47. Girard MJ, Suh J-KF, Bottlang M, et al. Biomechanical changes in the sclera of monkey eyes exposed to chronic IOP elevations. Investigative ophthalmology \& visual science 2011;52(8):5656-69.

48. Grytz R, Girkin CA, Libertiaux V, Downs JC. Perspectives on biomechanical growth and remodeling mechanisms in glaucoma. Mechanics research communications 2012;42:92106. 
49. Lee BL, Bathija R, Weinreb RN. The definition of normal-tension glaucoma. Journal of glaucoma 1998;7(6):366-71.

50. Mallick J, Devi L, Malik PK, Mallick J. Update on normal tension glaucoma. Journal of ophthalmic \& vision research 2016;11(2):204.

51. Shields MB. Normal-tension glaucoma: is it different from primary open-angle glaucoma? Current opinion in ophthalmology 2008;19(2):85-8.

52. Xie X, Chen W, Li Z, et al. Noninvasive evaluation of cerebrospinal fluid pressure in ocular hypertension: a preliminary study. Acta ophthalmologica 2018;96(5):e570-e6.

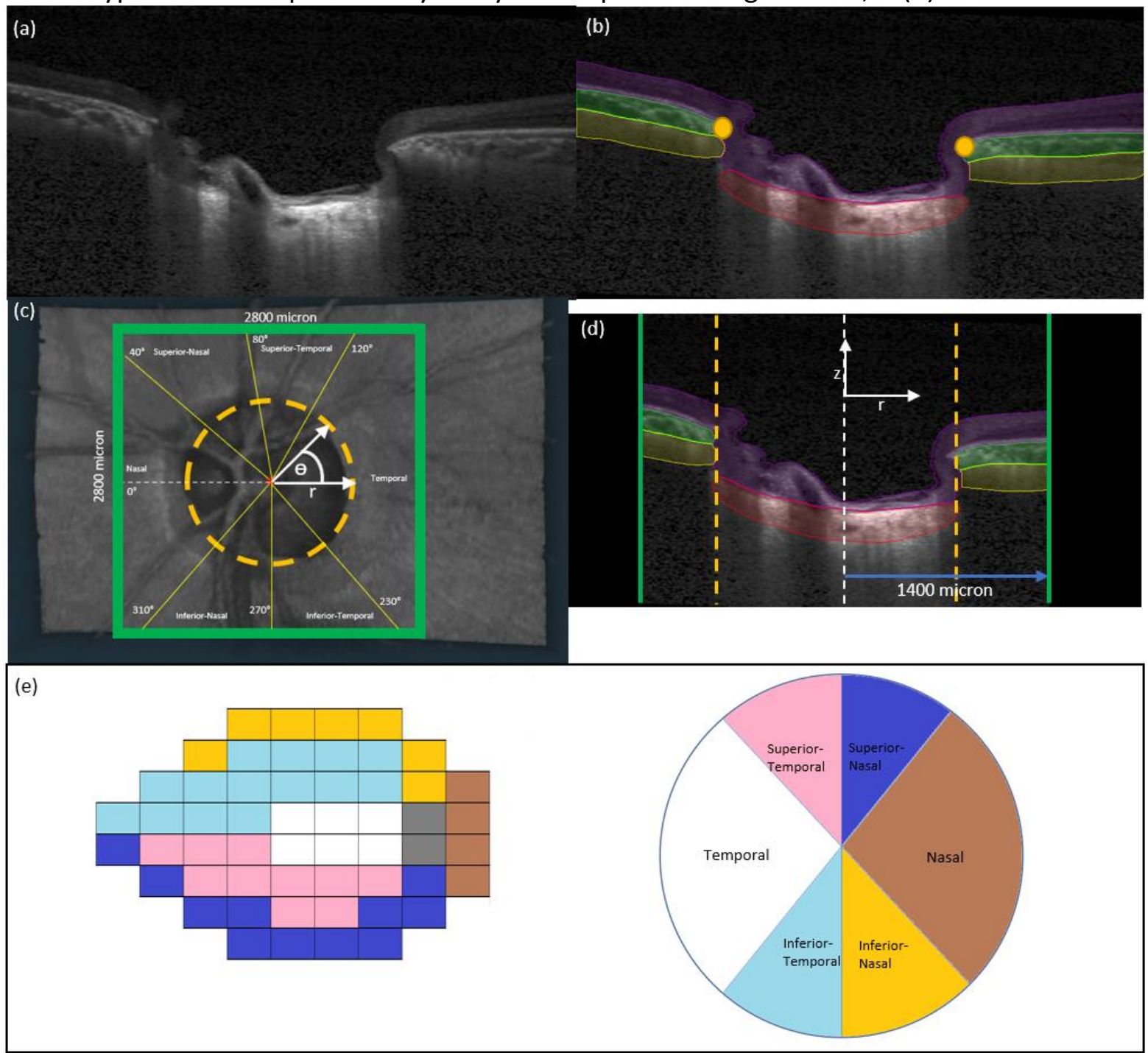

Figure 1(a) A single B-scan obtained from the OCT machine without any image enhancement (b) Automatic segmentation of the B-scan in (a). Four tissues were segmented - Pre-Lamina tissue (purple), Choroid (green), Sclera (yellow) and LC (red) In addition, BMOs (orange dots) were automatically marked for each B-scan (c) Anterior-surface view of the ONH. The ONH center (white star) was identified from the best-fit circle to the BMOs (orange-dotted line). Green square defines our region of interest to be cropped from the OCT volume with $2800 \mu m$ length on each side. Yellow lines divide the $\mathrm{ONH}$ into 6 based on the angular degree from the reference white-dotted line. White axes represent a cylindrical coordinate system ( $\Theta$ is circumferential direction, $r$ is radial direction). (d) A Bscan view after we apply cropping to the OCT volumes. Black region was not considered for our analysis. The length from central line (white-dotted line) to the cropping border (green line) is $1400 \mu \mathrm{m}$. White axes represent a cylindrical coordinate system ( $z$ is axial direction, $r$ is radial direction). (e) Regionalization of the 24-2 visual field test (left) to the ONH tissue (right) according to the GarwayHeath scheme. 
HTG

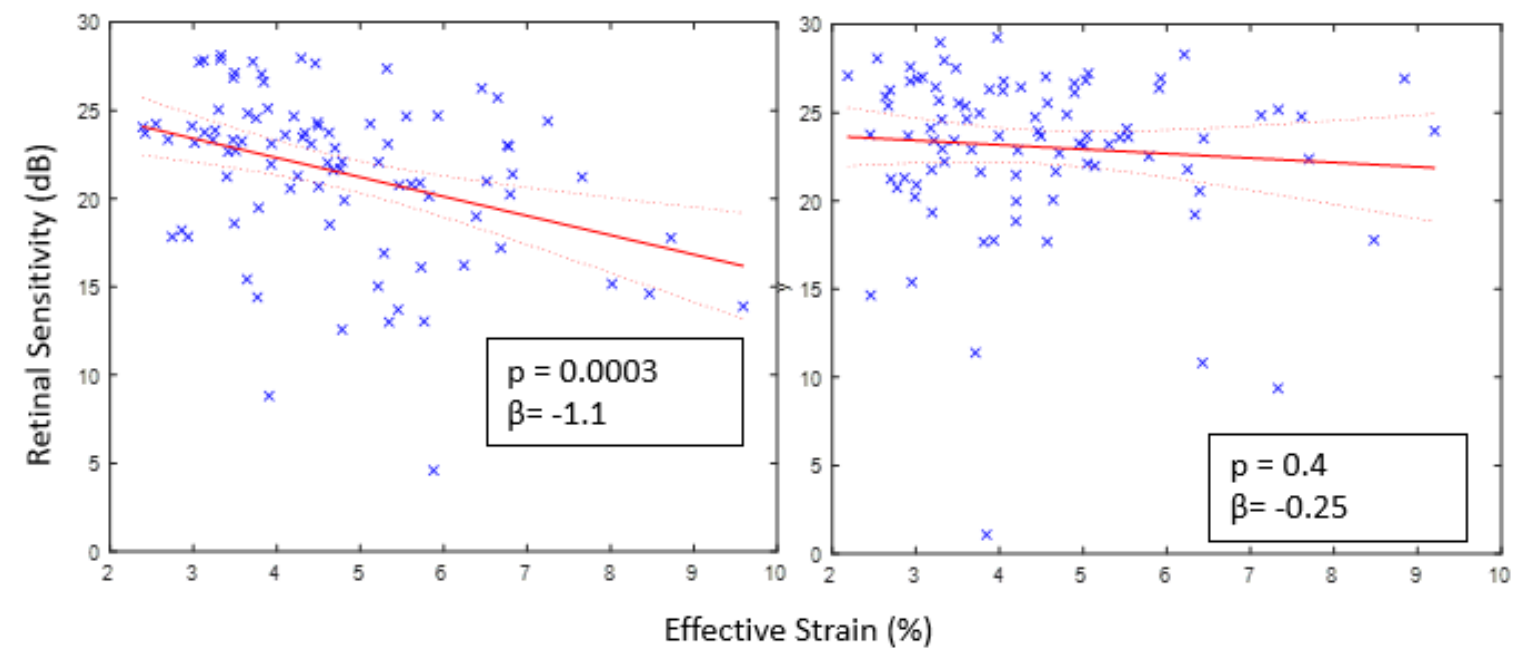

Figure 2 A scatter plot of retinal sensitivity $(\mathrm{dB})$ against effective strain $(\%)$ with regression lines (red) and confidence bounds (red-dotted) for HTG and NTG subjects. p represents p-value of the linear fit and $\beta$ represents the coefficient of the fit. 
(a)

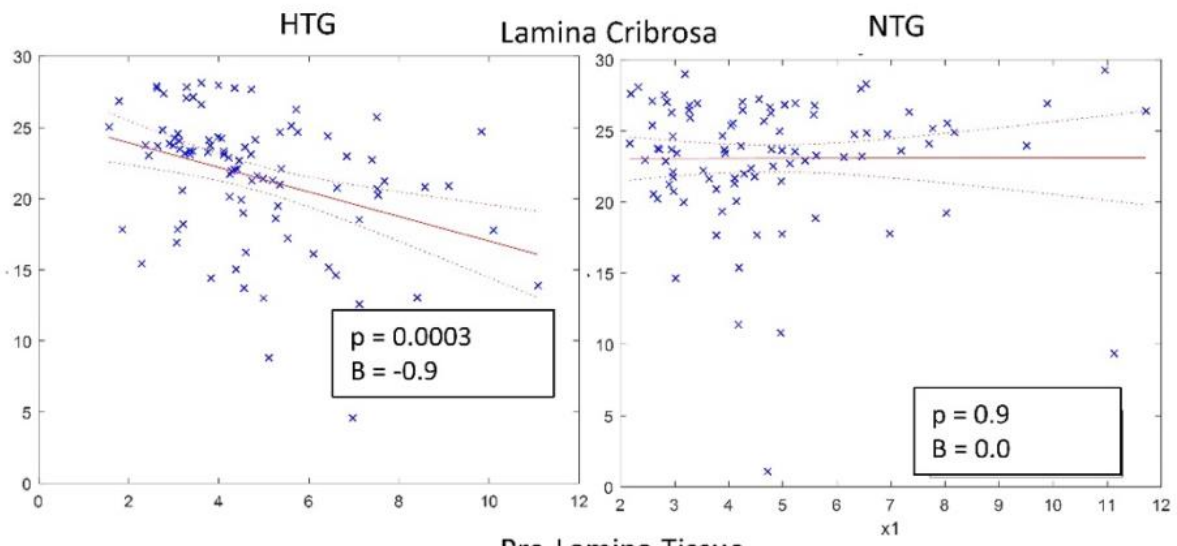

(b)

Pre-Lamina Tissue
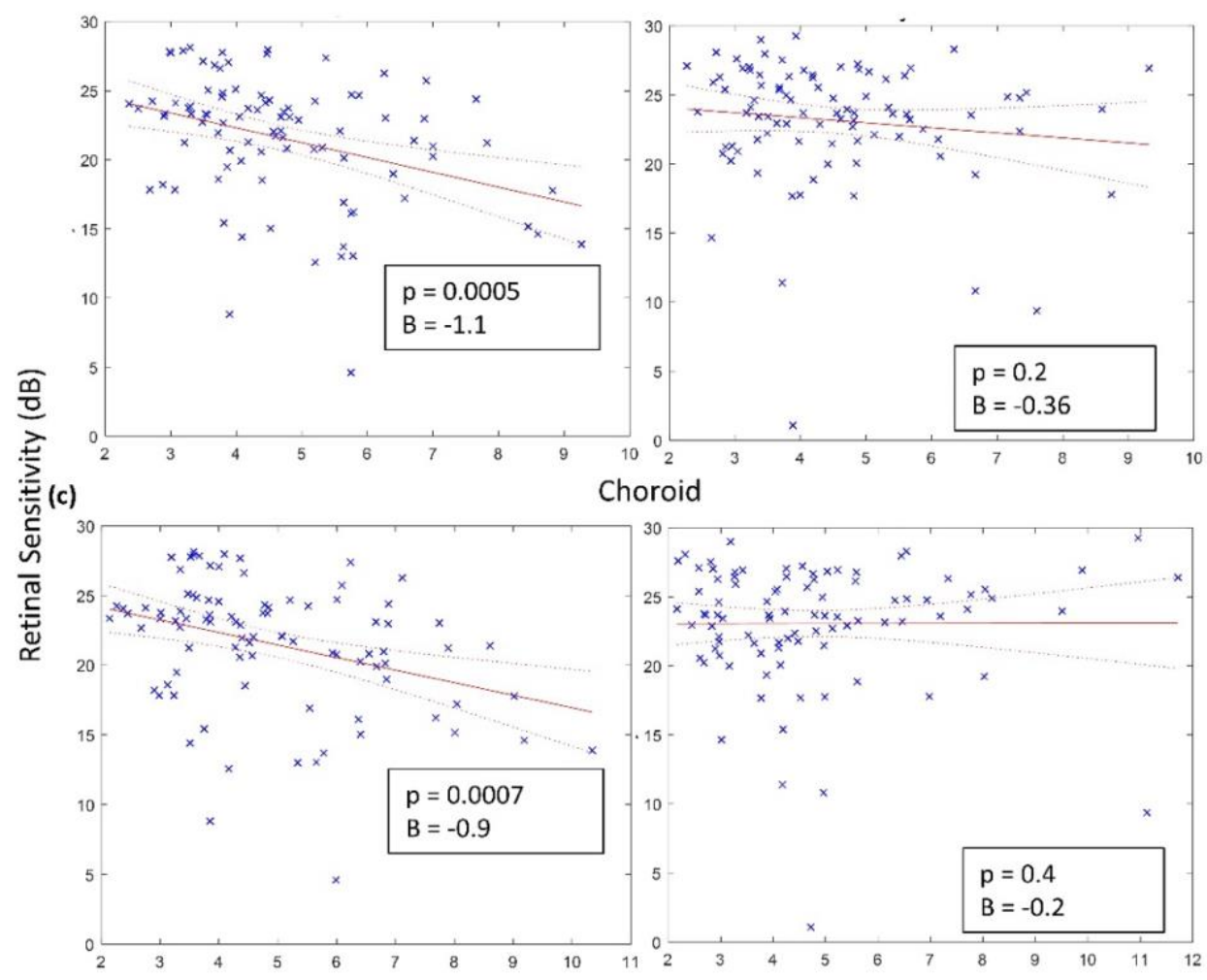

(d)

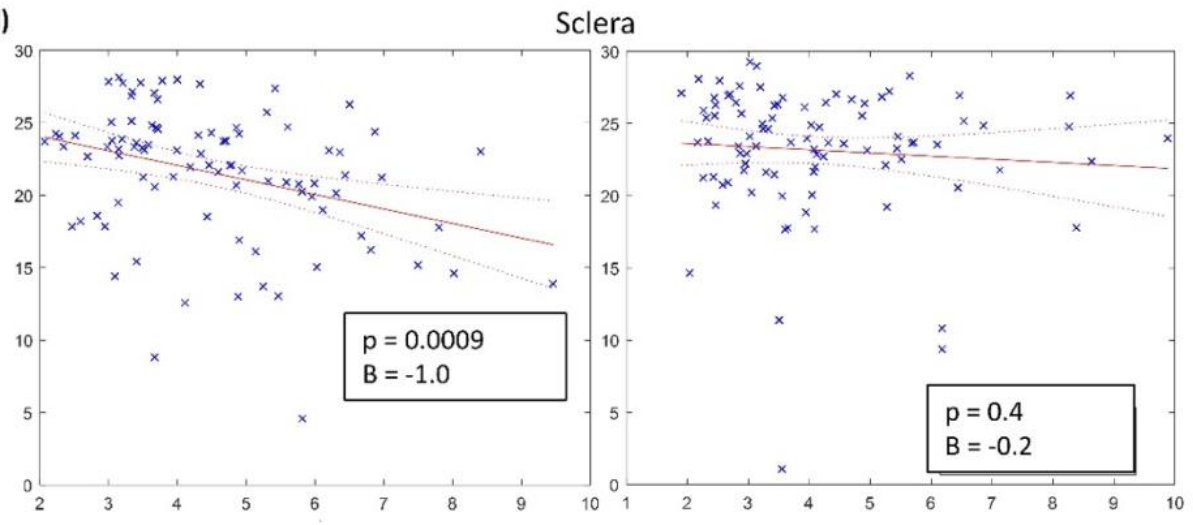

Effective Strain (\%)

Figure 3 A scatter plot of retinal sensitivity $(\mathrm{dB})$ against effective strain (\%) with regression lines (red) and confidence bounds (red-dotted) for the (a) lamina cribrosa (b) pre-lamina tissue (c) choroid and (d) sclera of the HTG and NTG subjects. $p$ represents $p$-value of the linear fit and $\beta$ represents the coefficient of the fit. 
(a)

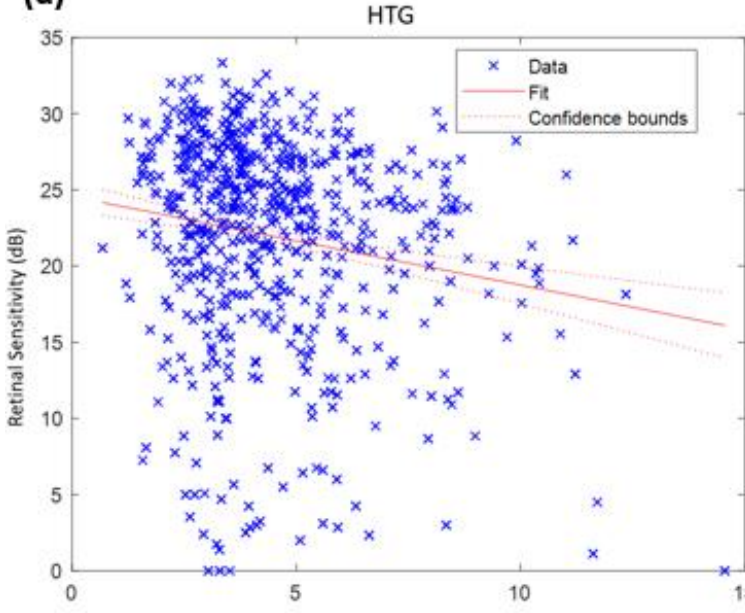

NTG

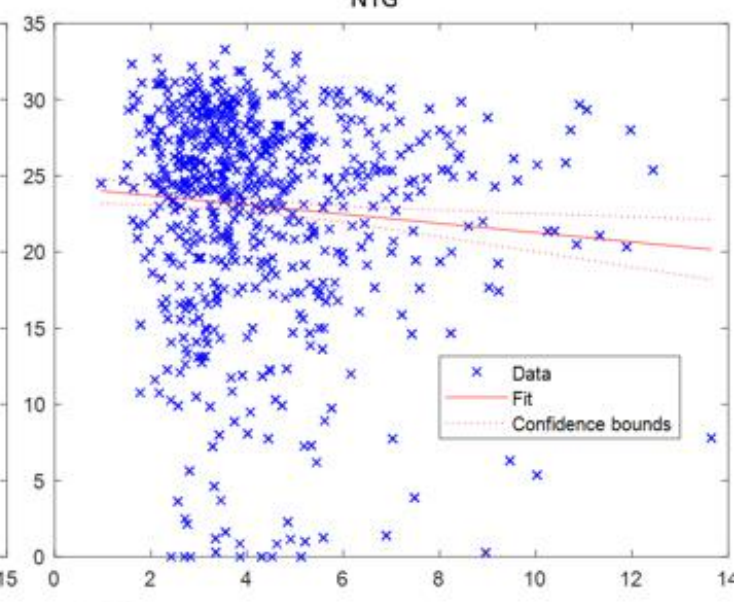

Effective Strain (\%)

HTG Subject
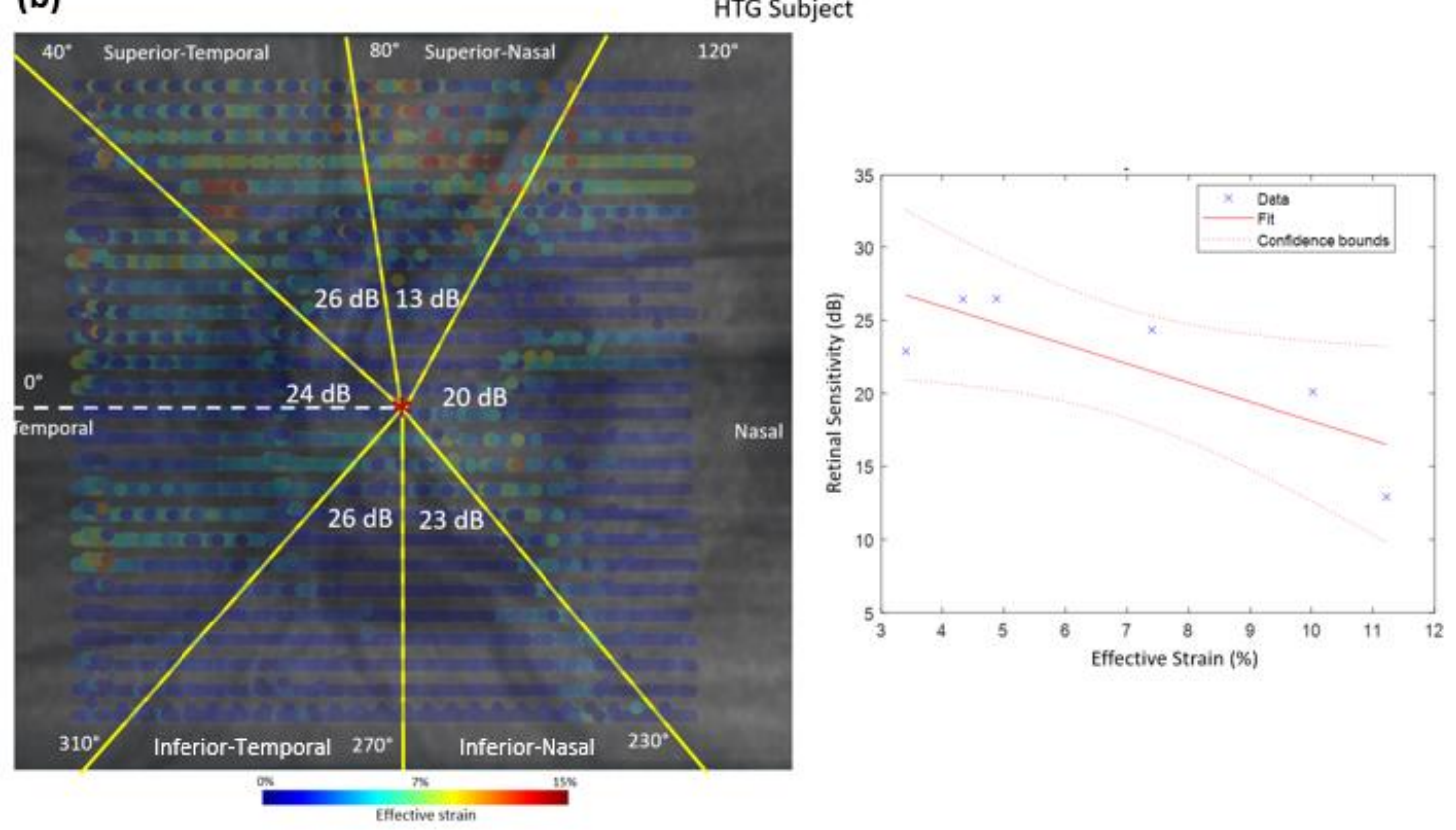

(c) NTG Subject
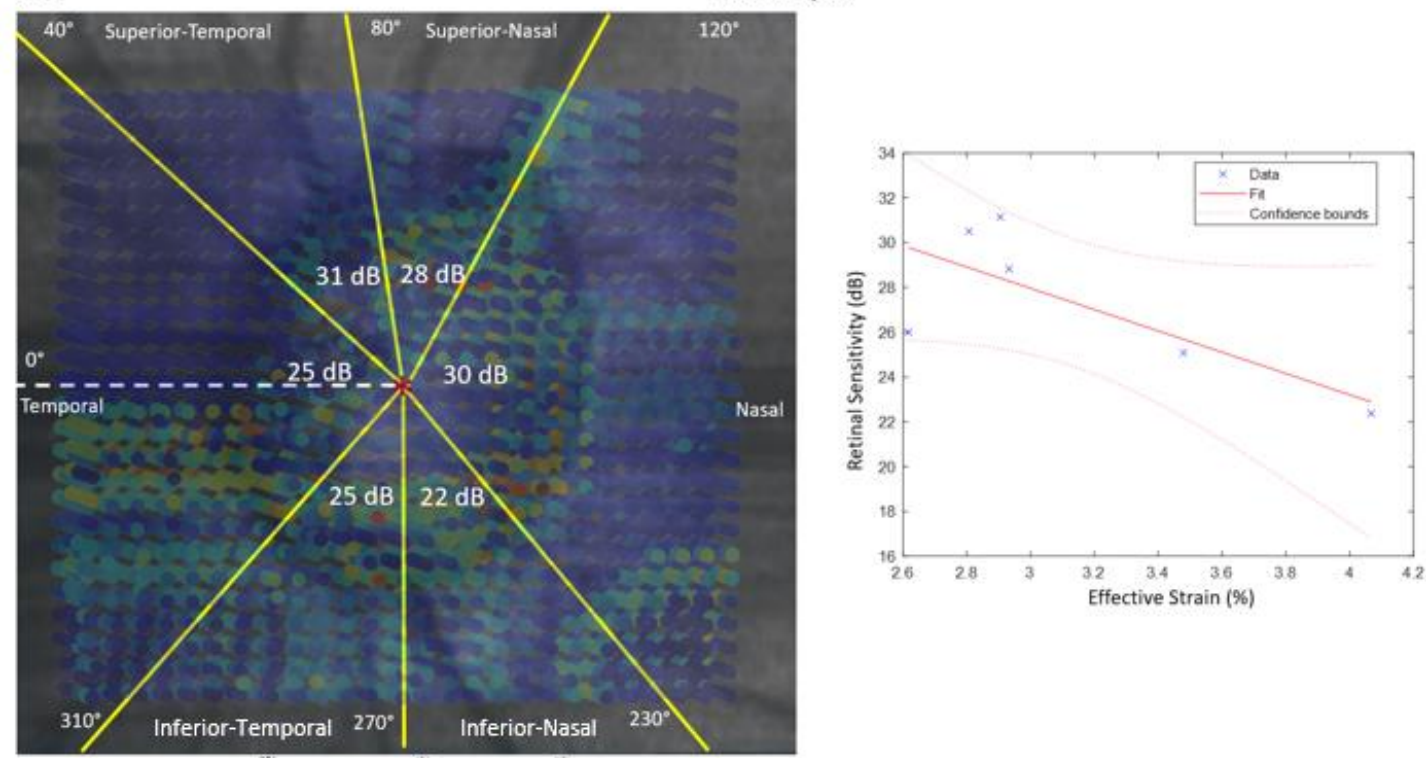

Effective strain 
Figure 4 (a) Scatter plots of all subjects (6 data points for each subject) of the average retinal sensitivity values against the effective strain. (b) Overlay of calculated effective strain on the en-face image of a single HTG subject's ONH (left) with corresponding colour bar to represent the strain values. Average retinal sensitivity values for each region are given in white text. Scatter plot (right) with linear regression line showing linear associations between average effective strain and average retinal sensitivity in each region. (c) Same representation as (a) for an NTG subject.

\begin{tabular}{|l|r|r|l|r|l|}
\hline Tissue & Subject Group & \multicolumn{1}{|l|}{ Region } & Strain & $\beta$ & P-value \\
\hline PLT & HTG & Superior-Temporal & Circumferential Strain & -3.65 & $5.86 \mathrm{E}-05$ \\
\hline PLT & HTG & Superior-Nasal & Circumferential Strain & -3.13 & $1.15 \mathrm{E}-05$ \\
\hline LC & NTG & Superior-Temporal & Circumferential Strain & -2.74 & $3.15 \mathrm{E}-05$ \\
\hline PLT & HTG & Superior-Temporal & Effective Strain & -1.65 & $1.54 \mathrm{E}-05$ \\
\hline Sclera & HTG & Superior-Temporal & Effective Strain & -1.26 & $1.74 \mathrm{E}-05$ \\
\hline Choroid & HTG & Superior-Temporal & Effective Strain & -1.04 & $4.89 \mathrm{E}-05$ \\
\hline
\end{tabular}

Table 1. Ranking of all significant associations (Bonferroni corrected from 142 total associations) between each type of strain and retinal sensitivity values with respect to each tissue type and each region. 